\section{Integrin-linked kinase controls vascular wall formation by negatively regulating Rho/ROCK-mediated vascular smooth muscle cell contraction}

\author{
Naoko Kogata, ${ }^{1,2}$ Rachel M. Tribe, ${ }^{3}$ \\ Reinhard Fässler, ${ }^{4}$ Michael Way, ${ }^{2,6}$ and \\ Ralf H. Adams ${ }^{1,5,7}$
}

${ }^{1}$ Vascular Development Laboratory, UK London Research
Institute, London WC2A 3PX, United Kingdom; ${ }^{2}$ Cell Motility
Laboratory, Cancer Research, UK London Research Institute,
London WC2A 3PX, United Kingdom; ${ }^{3}$ Maternal and Fetal
Research Unit, Division of Reproduction and Endocrinology,
King's College London, St. Thomas' Hospital Campus, London
SE1 7EH, United Kingdom; ${ }^{4}$ Max Planck Institute of
Biochemistry, 82152 Martinsried, Germany; ${ }^{5}$ Max-Planck-
Institute for Molecular Biomedicine, and University of Münster,
Faculty of Medicine, D-48149 Münster, Germany

Vascular smooth muscle cells (VSMCs) form contractile layers around larger blood vessels in a process that is essential for the formation of a fully functional vasculature. Here, we show that integrin-linked kinase (ILK) is required for the formation of a unitary layer of aligned VSMCs around arterioles and the regulation of blood vessel constriction in mice. In the absence of ILK, activated Rho/ROCK signaling induces the elevated phosphorylation of myosin light chain leading to abnormally enhanced VSMC contraction in vitro and in vivo. Our findings identify ILK as a key component regulating vascular wall formation by negatively modulating VSMC contractility.

Supplemental material is available at http://www.genesdev.org.

Received April 17, 2009; revised version accepted August 18, 2009.

The recruitment of pericytes and vascular smooth muscle cells (VSMCs), which form the outer layer of blood vessels, is a key process during angiogenesis and arteriovenous specification (Armulik et al. 2005). VSMCs, but not pericytes, envelope arteries and veins (Armulik et al. 2005). VSMCs also become highly elongated and aligned on small arteries (arterioles) to form a so-called unitary smooth muscle layer (Jakkaraju et al. 2003). The unitary layer is interconnected electronically and ionically with a nexus of the adjoining VSMCs and acts as a functional syncytium to maintain vascular tone, contractility, and vascular wall rigidity (Jakkaraju et al. 2003). Disorgani-

[Keywords: ILK; vascular wall formation; unitary layer; vascular smooth muscle cells (VSMCs); contractility; ROCK]

Correspondence authors.

${ }^{6}$ E-MAIL michael.way@cancer.org.uk; FAX 44-207-269- 3581.

${ }^{7}$ E-MAIL ralf.adams@mpi-muenster.mpg.de; FAX 49-251-70365-499.

Article is online at http://www.genesdev.org/cgi/doi/10.1101/gad.535409. zation of the unitary layer and loss of VSMC contractility is a feature often associated with atherosclerosis and other vascular diseases (Hao et al. 2003). However, the importance of the unitary layer in vascular development remains to be established.

Individual VSMCs in the unitary layer also secrete extracellular matrix and develop attachments to the basement membrane through integrin-associated cell adhesions (Moiseeva 2001). Integrins are $\alpha / \beta$ heterodimeric transmembrane receptors that link the extracellular matrix to the actin cytoskeleton (Hynes 2002). This cell-matrix connection also provides additional stability to the vessel wall (Moiseeva 2001). Genetic studies have revealed that the adaptor molecule integrin-linked kinase (ILK) is essential for integrin function in vivo (Delon and Brown 2007). In developing striated muscle, ILK is recruited to sarcomeric adhesion plaques where integrins bind to the extracellular matrix (Zervas et al. 2001). This recruitment is necessary for the assembly of myocytes into myotubes and the function of striated muscle (Zervas et al. 2001).

Previously, we reported that the major $\beta$ integrin subunit $\beta 1$ contributes to vascular smooth muscle function (Abraham et al. 2008). Gene inactivation of $\beta 1$ integrin in mice impairs VSMC spreading and differentiation, and increases VSMC proliferation leading postnatal lethality (Abraham et al. 2008). Here, we report that ILK plays a fundamental role in developing vascular wall assembly that is distinct from that of $\beta 1$ integrin. Mice lacking ILK expression in vascular wall cells fail to assemble their VSMCs into a unitary layer, which results in defective vascular remodeling and embryonic lethality. Moreover, we show that loss of ILK causes increased VSMC contractility due to elevated myosin light-chain phosphorylation through activation of Rho/ROCK signaling.

\section{Results and Discussion}

Defective vessel wall formation and embryonic lethality in $\mathrm{Ilk}^{\text {Pdgfrb-Cre }}$ mice

Since the early lethality of global Ilk knockout mice precludes studies in the vasculature, we bred mice carrying a loxP-flanked Ilk gene $\left(I l k^{f l / f 1}\right)$ and Pdgfrb-Cre mice to examine the consequences of Ilk gene deletion selectively in vascular wall cells. The offspring, herein referred to as Ilk ${ }^{\text {Pdgfrb-Cre }}$, resulted in a loss of ILK expression in dermal pericytes and developing VSMCs but not in endothelial cells (Supplemental Fig. 1A). Ilk ${ }^{\text {Pdgfrb-Cre em- }}$ bryos developed local hemorrhage and edema, which were first evident around embryonic day 13.5 (E13.5) (Fig. 1A; Supplemental Fig. 1B). Growth and organ morphogenesis also appeared delayed in late-stage $I 1 k^{\text {Pdgfrb-Cre }}$ embryos (E18.5), and no living mutants were obtained after birth (Fig. 1A; Supplemental Fig. 1B).

Characterization of the embryonic dermal vasculature by whole-mount immunostaining showed that arterioles of control littermates were continuously covered by VSMCs at E15.5 (Fig. 1B). In contrast, arteriolar VSMC coverage was incomplete in E15.5 Ilk ${ }^{\text {Pdgfrb-Cre mutants }}$ and further reduced at E18.5 (Fig. 1B,C). Ilk ${ }^{\text {Pdgfrb-Cre }}$ pericytes also had an unusual round morphology by E18.5 (Fig. 1C). Our observations suggest that the phenotype associated with the loss of ILK first manifests itself 
A $\quad E 13.5$

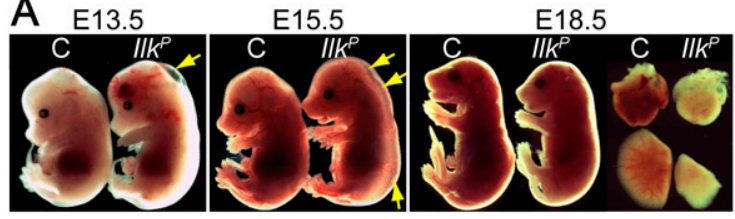

B

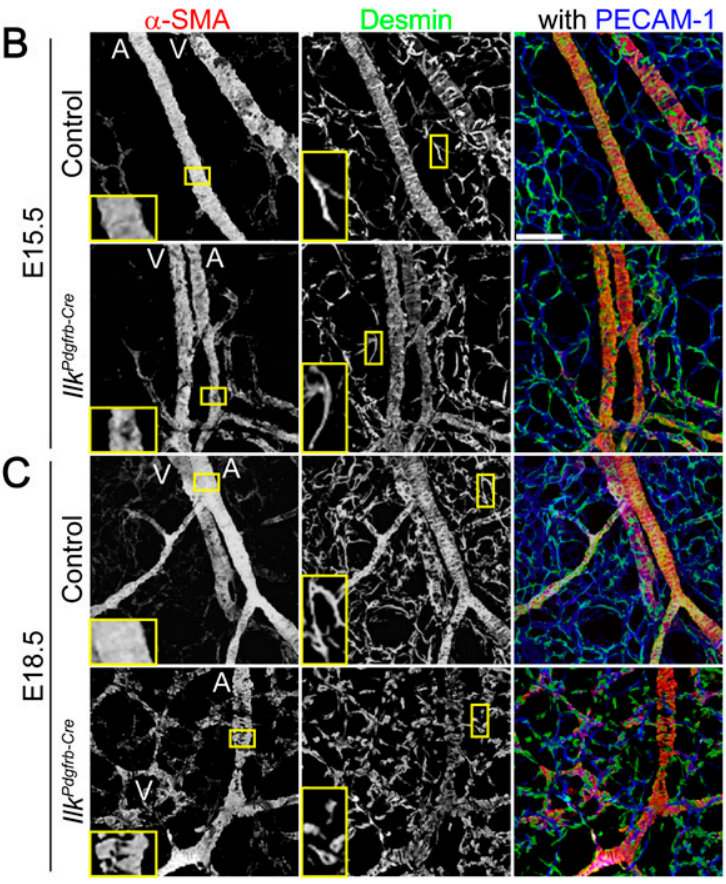

Figure 1. Impaired vascular wall formation and embryonic lethality in Ilk ${ }^{P d g f r b}$-Cre mice. (A) Gross examination of control (C) or $I l k^{\text {Pdgfrb-Cre }}\left(I l k^{P}\right)$ embryos at the indicated embryonic day (E). Arrows highlight edema. (Right) Heart and lung are shown to compare tissue morphogenesis. $(B, C)$ Confocal immunofluorescence images of whole-mount skin. VSMCs are labeled with $\alpha$-SMA, pericytes are desmin-only positive cells, and vascular endothelial beds are labeled with PECAM-1. (B, left, inset) At E15.5 ILKdeficient VSMCs are discontinuous on arterioles compared with the control. (Middle, inset) ILK-deficient pericytes have a similar appearance as control pericytes. (C) At E18.5 the coverage of ILKdeficient VSMCs on arterioles is diminished (left, inset), and knockout pericytes have spreading defects (middle, inset). (Right) Reduced veins and capillaries are observed in the knockouts. Bar, $50 \mu \mathrm{m}$. (A) Arteriole; (V) venule.

in VSMCs and later in pericytes. Furthermore, a wildtype-like parallel arrangement of arterioles and veins was seen at E15.5, but the venous architecture was highly disorganized by E18.5, suggesting blood vessel stability

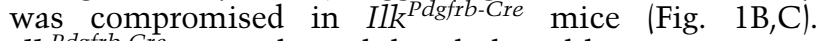
$I 1 k^{P d g f r b-C r e}$ mice also exhibited altered basement membrane assembly and minor VSMC apoptosis at E18.5 (Supplemental Fig. 1C,D). However, no overt change was detected in caspase activation or PKB/Akt phosphorylation in Ilk ${ }^{\text {Pdgfrb-Cre }}$ tissues (data not shown).

Lack of unitary smooth muscle layers in $\mathrm{Ilk}^{\text {Pdgfrb-Cre }}$ mice

To investigate the primary cause of reduced VSMC coverage in Ilk ${ }^{P d g f r b-C r e}$ mice, we examined the association of VSMCs with skin arterioles during early vascular wall formation at E13.5. Control VSMCs formed a unitary smooth muscle layer consisting of elongated and regularly orientated VSMCs (Fig. 2A). In contrast, ILKdeficient VSMCs failed to align, and were associated with arterioles in a disorganized fashion (Fig. 2A). Nevertheless, $\beta 1$ integrin was still concentrated between adjacent VSMCs of mutant mice, as seen in control littermates (Fig. 2A). Our in vivo observations indicate that ILK is required to organize VSMCs into the unitary smooth muscle layer.

It is generally thought that the increased transmural pressure within arterioles during maturation of the fetal circulation is responsible for promoting the alignment of VSMCs (Jakkaraju et al. 2003; Flavahan et al. 2005). To examine whether ILK is indeed necessary for the mechanoresponse to induce VSMC alignment, we subjected immortalized mouse control and ILK-deficient VSMCs to cyclic stretching in vitro. Control $I l k^{f l / f 1}$ VSMCs aligned perpendicular to the stretching axis and developed ventral actin stress fibers (Fig. 2B). In contrast, ILK-deficient $\left(I l k^{k o}\right)$ cells were less aligned and contained prominent cortical actin bundles (Fig. 2B). These data indicate that ILK is necessary to organize VSMC alignment in response to mechanical stretching.

\section{Loss of ILK induces matrix contraction and vessel constriction}

It is thought that ILK is involved in regulating extracellular matrix assembly by mediating an inside-out signaling cascade (Delon and Brown 2007). To investigate whether ILK deficiency affects the surrounding matrix,

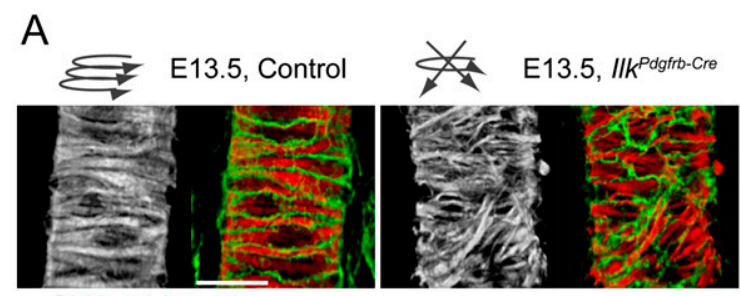

$\alpha$-SMA $\beta 1$ integrin
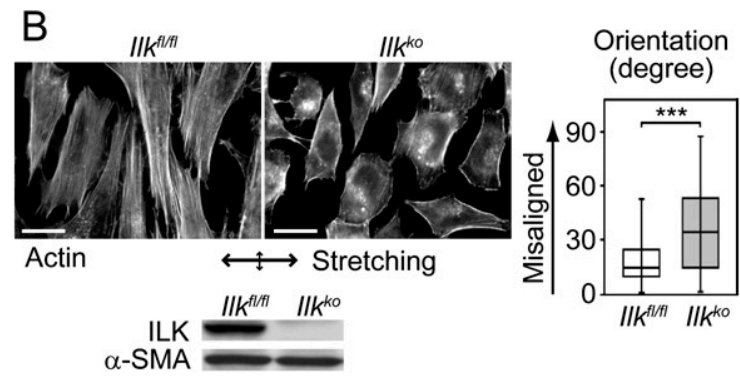

Figure 2. Failure in the formation of a unitary layer of ILK-deficient VSMCs. (A) Images of VSMCs labeled with $\alpha$-SMA and $\beta 1$ integrin at E13.5. (Right) ILK-deficient VSMCs lack uniform orientation on arterioles. Bar, $25 \mu \mathrm{m}$. (B) The effects of mechanical strain on the morphology of immortalized mouse VSMCs. (Left) Control Ilk $\mathrm{k}^{f l / f 1}$ VSMCs and their stress fibers become aligned vertical to the biaxial stretching. (Right) In contrast, the Ilk ${ }^{k o}$ VSMCs are less orientated and have prominent cortical actin rather than ventral stress fibers. The double-ended arrow indicates the direction of stretching. Bar, $20 \mu \mathrm{m}$. The degree of orientation of independent cells is summarized in the graph $(n=169)$. Western blotting confirms the depletion of ILK in Ilk ${ }^{k o}$ cells. 
we performed three-dimensional (3D) collagen matrix contraction assays using primary human VSMCs (hVSMCs). Examination of the 3D culture reveals that control hVSMCs were elongated and largely orientated in the same direction (Fig. 3A). In contrast, hVSMCs with reduced ILK expression following siRNA-mediated knockdown had a more chaotic arrangement that was reminiscent of that seen on blood vessels in Ilk ${ }^{\text {Pdgfrb-Cre }}$ mice (Figs. 2A, 3A; Supplemental Fig. 2). Interestingly, the loss of ILK resulted in a significant increase in the ability of hVSMCs to promote matrix contraction (Fig. $3 \mathrm{~B}, \mathrm{C})$. Consistent with the increased collagen matrix contraction in vitro, we found that both arterioles and venules in E15.5 knockout skin were significantly more constricted than those of control mice (Fig. 3D). Thus, the presence of ILK limits vascular constriction, thereby facilitating normal vessel wall development.

Loss of ILK modulates VSMC contractility and focal adhesion assembly

In culture, $I 1 k^{k o}$ VSMCs were more contracted than control cells (Fig. 4A,B). Moreover, phalloidin-positive actin stress fibers in knockout VSMCs were mainly

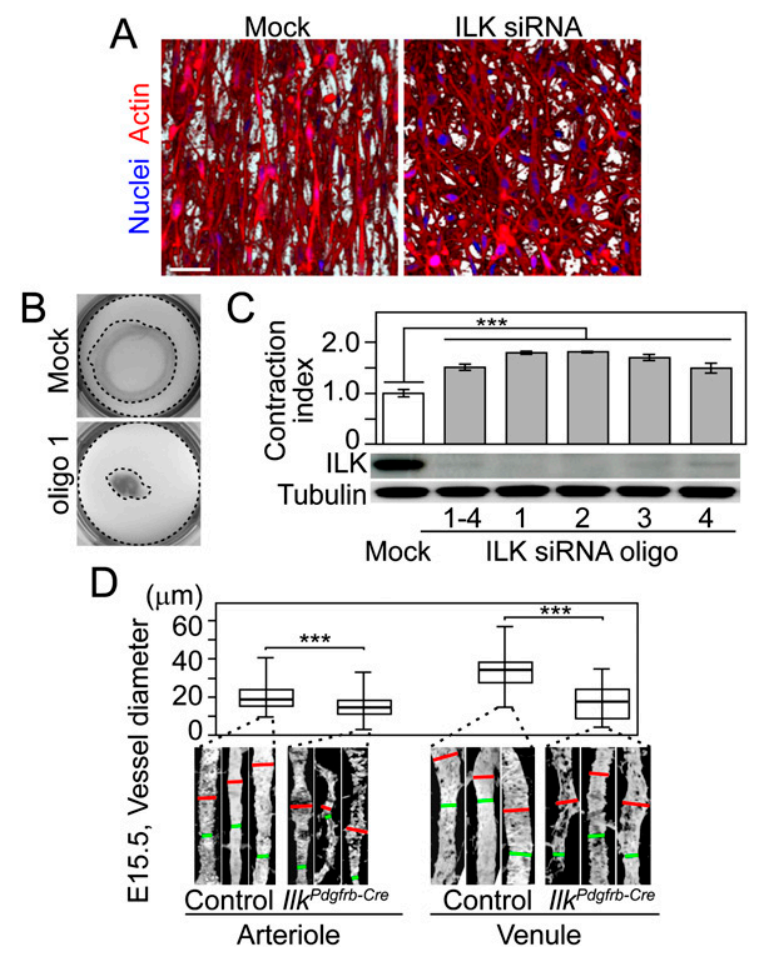

Figure 3. Extracellular matrix contraction and vessel constriction facilitated by ILK-deficient VSMCs. (A) Images of hVSMC visualized with phalloidin reveal that mock-treated hVSMCs are regularly arranged, while ILK knockdown hVSMCs have random orientation. Bar, $50 \mu \mathrm{m}$. (B) Representative image of increased gel contraction (1.79 \pm 0.03 times more than control) induced by hVSMCs treated with ILK siRNA oligol. The outer and inner dotted lines indicate the area of the initial and contracted gel, respectively. $(C)$ Quantification of gel contraction $(n=7)$ induced by hVSMCs treated with the indicated ILK siRNA oligos together with the corresponding Western blot showing the knockdown of ILK. (D) Quantification of blood vessel diameter at E15.5. The diameter is calibrated manually using Volocity software on the widest (red) and narrowest (green) points of a single vessel ( $n=88$ from seven mice).

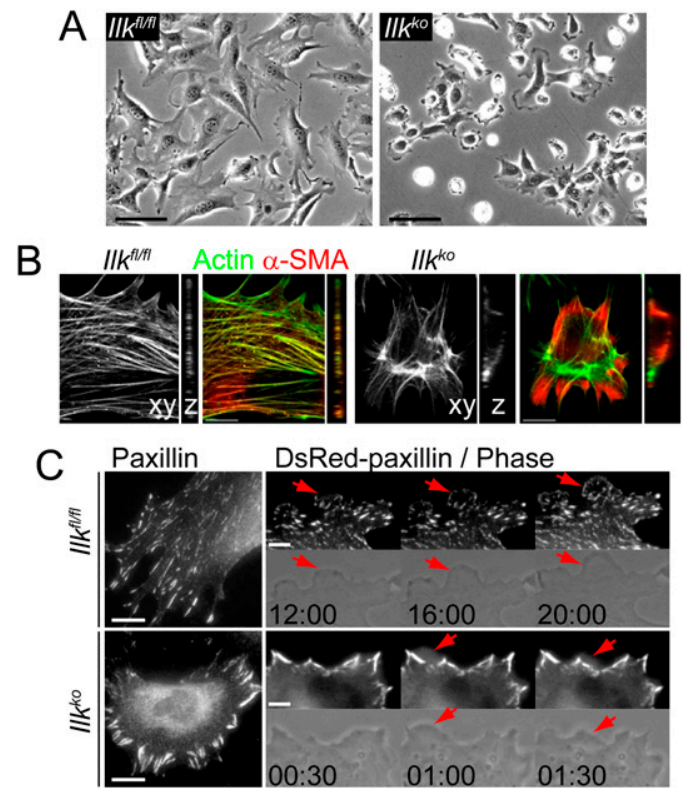

Figure 4. Loss of ILK increases cell contractility and changes focal adhesion assembly. (A) Phase images showing that loss of ILK results in defects in VSMC spreading. Bar, $50 \mu \mathrm{m}$. $(B)$ Confocal plane $(\mathrm{xy})$ and vertical view $(z)$ of $\alpha$-SMA and actin cytoskeleton reveal that actin fibers in $I l k^{k o}$ cells are concentrated within the cell rather than being distributed throughout the cell. Bar; $10 \mu \mathrm{m}$. (C) Paxillin immunostaining reveals that focal adhesions are enlarged and principally observed at cell periphery in Ilk ${ }^{k o}$ VSMCs. Bar, $25 \mu \mathrm{m}$. The fluorescent and phase panels from time-lapse movies show the formation and dissociation of nascent focal adhesions at the expanding lamellipodia (red arrows). In contrast to migrating control cells, Ilk ${ }^{k o}$ VSMCs, which remain stationary, undergo constant ruffling and fail to establish focal complexes (red arrows). The time in minutes and seconds is indicated in each panel. Bar, $5 \mu \mathrm{m}$.

locally concentrated rather than being evenly distributed throughout the cell (Fig. 4B). These dramatic differences in cell morphology are due to an absence of ILK, as Ilk ${ }^{k o}$ VSMCs expressing ILK-EGFP were spread and had actin stress fibers distributed throughout the cell (Supplemental Fig. 3).

Consistent with the differences in cell spreading and contractility, the organization of focal adhesions was also affected by the loss of ILK. The absence of ILK, however, did not affect cell adhesion on collagen-I or fibronectin (Supplemental Fig. 4). Live-cell imaging revealed that, in migrating control cells, lamellipodia extended and retracted over a period of several minutes, concomitant with the formation and dissociation of paxillin-positive focal complexes at the protrusive edge (Fig. 4C; Supplemental Movies 1, 3). However, Ilk ${ }^{k o}$ cells showed little or no migration and displayed constitutive ruffling without forming paxillin-positive focal complexes (Fig. 4C; Supplemental Movies 2, 4). Consistent with the decreased migration, the center of $I l k^{k o}$ cells contained very few focal adhesions (Fig. 4C). ILK-deficient cells, however, developed large peripheral focal adhesions behind lamellipodia, a region where the retrograde flow of actin is generated by myosin II-dependent contractility (Fig. 4C; Supplemental Movie 4). This is consistent with previous reports that the size of focal adhesions behind lamellipodia correlates with the contractility of the cell (Giannone et al. 2007). Our observations suggest that ILK regulates 
VSMC contractility and focal adhesion assembly during cell migration.

\section{Rho/ROCK signaling increases myosin light chain (MLC) phosphorylation in the absence of ILK}

Smooth muscle cell contractility is dependent on the activity of myosin II, which is regulated by the status of MLC phosphorylation (Webb 2003). Consistent with their increased contractility in vitro, we found that $I 1 k^{k o}$ VSMCs had elevated levels of MLC phosphorylation (Fig. 5A,B). In smooth muscle, MLC phosphorylation is principally controlled by two kinases. MLC kinase (MLCK) activates myosin II by directly phosphorylating MLC (Webb 2003). In contrast, the RhoA effector ROCK can phosphorylate myosin phosphatase target subunit 1 (MYPT1) at Thr853 to promote the dissociation of the phosphatase complex from myosin II (Velasco et al. 2002). It has also been reported that ROCK can directly phosphorylate MLC in vitro (Amano et al. 1996).

To determine which pathway is responsible for increasing MLC phosphorylation in the absence of ILK, we examined the consequences of treating Ilk ${ }^{k o}$ VSMCs with inhibitors of MLCK or ROCK. Inhibition of MLCK with ML-9 had no obvious effect on MLC phosphorylation and did not induce spreading of $I l k^{k o}$ VSMCs (Fig. 5B,C). In contrast, inhibition of ROCK with Y-27632 significantly reduced MLC phosphorylation in $I 1 k^{k o}$ cells (Fig. 5A,B). The inhibition of ROCK in $I l k^{k o}$ VSMCs also resulted in a reduction in MYPT1 phosphorylation, the redistribution of actin stress fibers, and increased cell spreading (Fig. 5A-C). Live-cell imaging revealed that inhibition of ROCK also suppressed the rapid membrane ruffling of $I 1 k^{k o}$ VSMCs concomitantly with the formation of ventral focal adhesions as cells initiate migration (Supplemental Movie 5). Expression of dominant-negative
ROCK1 or ROCK2 was also capable of reducing MLC phosphorylation and promoting spreading of $I l k^{k o}$ VSMCs (Supplemental Fig. 5). In addition, Ilk ${ }^{k o}$ VSMCs treated with Y-27632 also recovered the ability to orientate during mechanical stretching (Fig. 5D).

Consistent with the increased contractility, the level of active GTP-bound RhoA was also significantly elevated in $I 1 k^{k o}$ VSMCs, although the expression level of RhoA did not change (Fig. 5E; data not shown). Moreover, inhibition of RhoA signaling using the membrane-permeable TAT-C3 inhibitor was also able to reduce MLC phosphorylation and rescued the spreading of $I 1 k^{k o}$ VSMCs (Fig. 5B,C). The negative regulation of MLC phosphorylation by ILK is not restricted to VSMCs in culture. Immunostaining on tissue sections reveals that the absence of ILK also led to increased MLC phosphorylation in VSMCs associated with dermal arterioles of Ilk ${ }^{\text {Pdgfrb-Cre }}$ mice (Fig. 5F).

We demonstrated that ILK-controlled vascular wall coverage is essential during artery/vein specialization and normal embryonic development. This observation strongly supports previous findings in PDGF-B/PDGFR- $\beta$ knockout mice that vascular wall immaturity destabilizes the vascular network and impairs angiogenic remodeling (Hellstrom et al. 1999). The genetic inactivation of PDGF-B/PDGFR- $\beta$ or ILK results in embryonic lethality around E18.5, although they contribute to vascular wall formation in distinct ways. Unlike PDGF-B/PDGFR $\beta$ signaling, ILK does not appear to control the recruitment of pericytes to nascent vessels, but modulates subsequent steps of vessel wall maturation.

As mentioned above, ILK, but not $\beta 1$ integrin, is important for the formation of a functional unitary layer. Consistent with this difference, we found that VSMCs lacking $\beta 1$ integrin do not have altered levels of MLC phosphorylation or increased matrix contraction in vitro
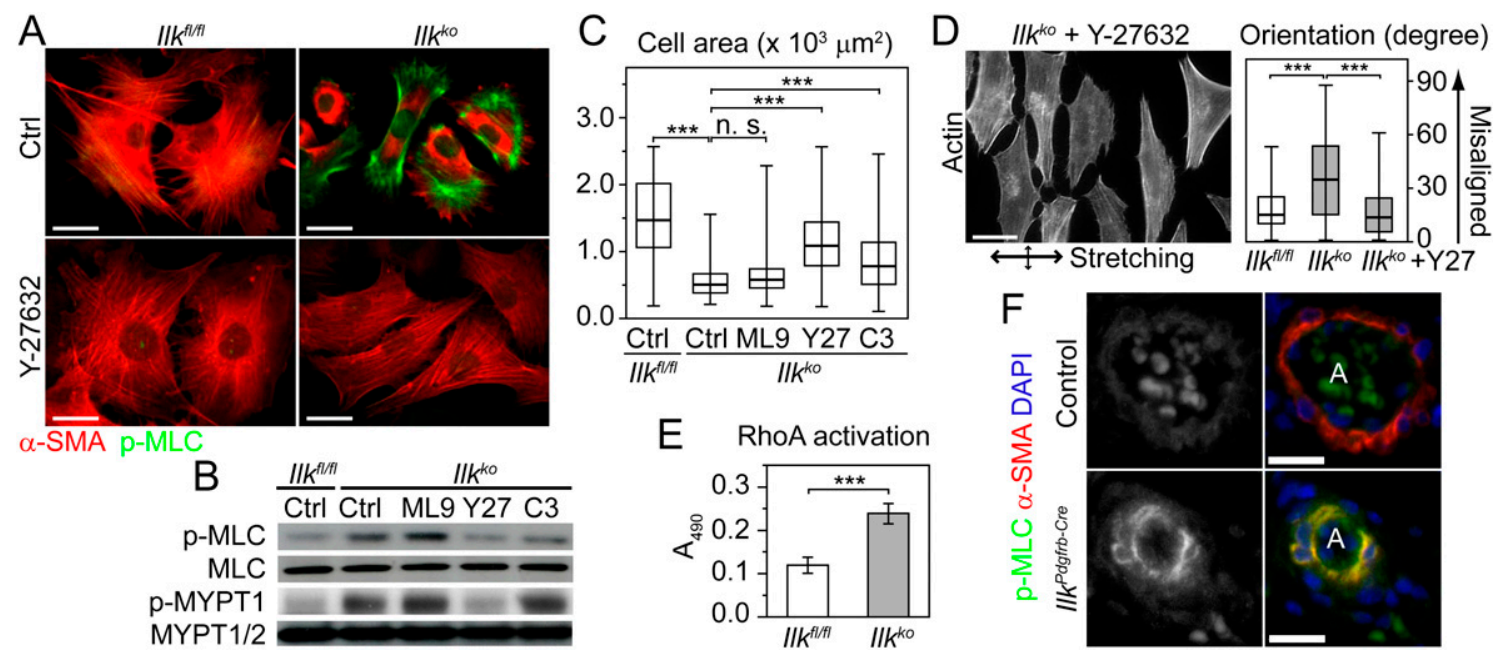

Figure 5. Increased MLC phosphorylation by activated ROCK signaling in the absence of ILK. (A) Immunostaining with the indicated antibodies demonstrates that MLC phosphorylation is dramatically increased in Ilk ${ }^{k o}$ cells. Addition of the ROCK inhibitor Y-27632 suppressed the increased MLC phosphorylation and promoted the redistribution of actin stress fibers and cell spreading. Bar, $25 \mu \mathrm{m}$. $(B)$ Western blot analysis reveals that phosphorylation of MLC (p-MLC: T18/S19) and MYPT1 (p-MYPT1: T853) are significantly increased in Ilk ${ }^{k o}$ cells. Inhibition of ROCK with Y-27632 (Y27) and RhoA with TAT-C3 (C3) but not MLC kinase (ML9) reduced phosphorylation of MLC in Ilk ${ }^{k o}$ VSMCs. (C) Inhibition of ROCK (Y27) and RhoA (C3) but not MLC kinase (ML9) also promoted increased spreading of $I 1 k^{k o}$ VSMCs $(n=297$ for each condition). (n.s.) No significance. (D) Immunofluorescence analysis reveals that mechanical stretching of $I l k^{k o}$ VSMCs in the presence of Y-27632 (Y27) results in increased alignment $(n=412)$. Bar; $20 \mu \mathrm{m}$. (E) Loss of ILK increases RhoA activation in VSMCs $(n=4)$. $(F)$ Immunofluorescence analysis of E15.5 skin (nine sections from seven mice) with the indicated antibodies reveals that loss of ILK results in increased MLC phosphorylation in VSMCs associated with arterioles in Ilk ${ }^{\text {Pdgfrb-Cre }}$ mice. Bar, $20 \mu \mathrm{m}$. 
(Supplemental Fig. 6). The difference in the vascular wall phenotype of mice lacking ILK and $\beta 1$ integrin presumably reflects the ability of ILK to interact with additional $\beta$ integrins (Legate et al. 2006; McDonald et al. 2008).

Previous biochemical analyses in vitro have suggested that ILK phosphorylates MLC and MYPT1 to promote VSMC contraction (Muranyi et al. 2002; Wilson et al. 2005). Nevertheless, there is considerable debate regarding whether ILK is actually a functional Ser/Thr kinase (Boudeau et al. 2006; Legate et al. 2006). Our observations in vivo and in vitro suggest that ILK negatively regulates VSMC contractility via Rho/ROCK-mediated inactivation of MYPT1 rather than directly phosphorylating MLC. This suggestion is consistent with recent observations in the nervous system (Pereira et al. 2009). Taken together, our collective data suggest that ILK is likely to play a universal role in negatively regulating Rho/ROCK signaling in a variety of cell types. The contracted morphology of $I l k^{k o}$ VSMCs also resembles that of embryonic fibroblasts or chondrocytes lacking a functional ILK gene as well as endothelial cells in which ILK expression was reduced by siRNA (Grashoff et al. 2003; Sakai et al. 2003; Vouret-Craviari et al. 2004). Our characterization of tissuespecific mutant mice and cultured knockout VSMCs firmly place ILK upstream of Rho/ROCK and show that it is a crucial regulator of VSMC contractility. Moreover, this essential role of ILK in vascular morphogenesis suggests that it may also play a role in disease conditions in which VSMC contractility is lost or deregulated. The task ahead is to determine the molecular mechanism by which ILK attenuates Rho/ROCK signaling.

\section{Materials and methods}

\section{Antibodies}

$\alpha$-Smooth muscle actin ( $\alpha$-SMA, clone 1A4), MLC (clone MY-21), and $\alpha$-tubulin (clone B-5-1-2) were from Sigma-Aldrich; PECAM-1 (clone MEC 13.3) and paxillin (clone 165) were from BD Biosciences; desmin and MYPT1/2 (clone YE336) were from Abcam; ILK (clone 65.1.9), $\beta 1$ integrin (clone MB1.2), collagen type IV, and pT853-MYPT1 (clone SA19) were from Millipore; and pT18/S19-MLC were from Cell Signaling Technologies. Secondary antibodies coupled with either Alexa dyes or HRP were purchased from Invitrogen and GE Healthcare, respectively.

\section{Mice and immunohistochemistry}

All animal experiments were approved by the Cancer Research UK Animal Ethics Committee. Pdgfrb-Cre mice and mice carrying a loxPflanked Ilk gene $\left(I l k^{f l / f 1}\right)$ were bred to obtain mutant $I 1 k^{\text {Pdgfrb-Cre }}$ and control $I^{\prime} k^{f l / f 1}$ embryos as controls in a mixed 129SV/C57BL6 genetic background (Grashoff et al. 2003; Foo et al. 2006). For mouse VSMC isolation, immorto $I 1 k^{f 1 / f 1}$ mice harboring temperature-sensitive SV40 large T-antigen were obtained as described previously (Foo et al. 2006). All progeny were genotyped by PCR prior to further analyses.

Antibody stainings of whole-mount tissue and skin paraffin sections were performed as described previously (Foo et al. 2006). All images were collected using an upright LSM510 confocal microscope (Carl Zeiss) and reconstituted using Volocity software (Improvision).

\section{Mouse VSMC isolation, culture, and immunofluorescence}

The isolation and culture of VSMCs was performed as described previously with a few modifications (Foo et al. 2006). To obtain immortalized Ilk ${ }^{k o}$ VSMCs, a cre cDNA-contained plasmid (pBS598; a gift from B. Sauer) was transiently transfected into $I I k^{f l / f 1}$ cells, and $I l k^{k o}$ cells were selected with $0.5 \mathrm{mg} / \mathrm{mL}$ Geneticin for $2 \mathrm{wk}$. To obtain a homogeneous cell line, 21 individual clones were tested by immunoblotting to confirm gene deletion of IIk as well as by the changes in cell morphology. As all 21 clones displayed a similar contracted cell shape, a single clone was expanded and used for all subsequent experiments. Smooth muscle identity was confirmed by the immunoreactivity for $\alpha$-SMA and SM $22 \alpha$. Immunofluorescence was performed on cells plated onto glass coverslips coated with rat tail type I collagen-coated (BD Biosciences). Images were obtained on an inverted Zeiss Axiovert 200M microscope (Carl Zeiss) using a Hamamatsu ORCA-ER camera (Hamamatsu Photonics) controlled by Volocity software.

\section{Application of cyclic strain}

Mouse VSMCs were plated onto rubber-bottom BioFlex Culture Plates coated with type I collagen (Flexcell International Corp.) at a density of $3 \times 10^{5}$ cells per well. After $48 \mathrm{~h}$, subconfluent cells were subjected to equibiaxial cyclic strain under computer-controlled vacuum device FX4000T with the following parameters: $10 \%-16 \%$ amplitude, $1 \mathrm{~Hz}$ constant frequency, and 18-h exposure. The stretched cells were fixed with $4 \%$ paraformaldehyde in PBS for $10 \mathrm{~min}$ and subsequently labeled with phalloidin (Invitrogen). Using Integrated Morphometry Analysis program on MetaMorph software (Molecular Devices), the angle of cell orientation was automatically measured to determine absolute scores up to $90^{\circ}$. The frequency of cell alignment was determined from (the number of aligned cells)/(total cell number).

\section{SiRNA and gel contraction assay}

Human VSMCs (six to nine passages) at $70 \%$ confluence were transfected with siRNA duplexes using Lipofectamine 2000 (Invitrogen). A mock experiment was performed on cells transfected with $10 \mathrm{nM}$ control BLOCKiT Fluorescent Oligo (Invitrogen). ILK knockdown was performed on cells transfected with $10 \mathrm{nM}$ BLOCK-iT and $20 \mathrm{nM}$ ON-TARGET plus human Ilk siRNA oligos1-6. Oligol (5'-GGGCACGGAUCAAUGUAAU-3'), oligo2 (5'-CAAUAGCCGUAGUGUAAUG-3'), oligo3 (5'-CGACCCAAA UUUGACAUGA-3'), and oligo4 (5'-GCACCAAUUUCGUCGUGGA-3') were obtained from Dharmacon. Oligo5 (5'-GGAAGAGCAGGGACU UCAATT-3') and oligo6 (5'-CAGCCCGAGTCCCGAGGATAA-3') were obtained from Qiagen.

Knockdown of ILK was validated by Western blotting from days 2 to 6 after the transfection. After $3 \mathrm{~d}$, cells were suspended at $0.5 \times 10^{6}$ cells per milliliter in $1.0 \mathrm{mg} / \mathrm{mL}$ type I collagen, $8 \%$ FBS, and $0.8 \times$ DMEM adjusted to the appropriate $\mathrm{pH}$ with $0.1 \mathrm{M} \mathrm{NaOH}$. The cell-matrix mixture was dropped onto BSA-coated 24-well plates with culture medium on the top to induce free contraction. After $30 \mathrm{~h}$, the pixel area of the gels was measured using ImageJ for quantitative evaluation. Contraction index was calculated from (well area - gel area)/(well area) and then normalized to the control. The contracted gels were fixed with $4 \%$ paraformaldehyde in PBS for $30 \mathrm{~min}$ and subsequently labeled with phalloidin and DAPI.

\section{Time-lapse imaging}

To trace the dynamics of integrin-based adhesions, DsRed-Paxillin was transfected into VSMCs on type I collagen-coated 35-mm glass-bottom dish (MatTek Co.) using an Amaxa Nucleofector device (Amaxa). Images were obtained every $30 \mathrm{sec}$ on an inverted Nikon TE2000-E (Nikon Instruments) using an Andor Ixon single-photon CCD camera controlled by MetaMorph.

\section{Inhibitor treatment and RhoA activation assay}

Mouse VSMCs were plated at 50\% confluence and, the next day, cells were treated with DMSO (for mock), $10 \mu \mathrm{M}$ ML-9, or $5 \mu \mathrm{M}$ Y-27963 (Calbiochem) for $6 \mathrm{~h}$, or TAT-C3 for $18 \mathrm{~h}$, and subjected to immunostaining or automatic cell area measuring as described previously (Foo et al. 2006).

Quantification of RhoA activation was performed using G-LISA RhoA Activation Assay Biochem Kit following the manufacturer's instruction (Cytoskeleton, Inc.).

\section{Statistical analysis}

The data in the graphs are presented as mean and standard deviation (SD) of the mean. Box and whisker plots are generated using median, quartiles, 
and highest and lowest values. The data were analyzed by two-tailed ANOVA or Student's $t$-test using Excel software. A $P$-value of $<0.05$ is considered statistically significant. Three asterisks $\left({ }^{\star \star \star}\right)$ indicate $P<$ 0.001 .

\section{Acknowledgments}

We thank Dr. Erik Sahai (Cancer Research UK) for DsRed-paxillin plasmid, Cell Motility laboratory members for advice on the manuscript, and members of the Biological Resources and Light Microscopy units for technical assistance. This work was supported by Cancer Research UK (N.K., M.W, and R.H.A.) and the Collaborative Research Centre 492 of the DFG (R.H.A.).

\section{References}

Abraham S, Kogata N, Fassler R, Adams RH. 2008. Integrin $\beta 1$ subunit controls mural cell adhesion, spreading, and blood vessel wall stability. Circ Res 102: 562-570.

Amano M, Ito M, Kimura K, Fukata Y, Chihara K, Nakano T, Matsuura Y, Kaibuchi K. 1996. Phosphorylation and activation of myosin by Rhoassociated kinase (Rho-kinase). J Biol Chem 271: 20246-20249.

Armulik A, Abramsson A, Betsholtz C. 2005. Endothelial/pericyte interactions. Circ Res 97: 512-523.

Boudeau J, Miranda-Saavedra D, Barton GJ, Alessi DR. 2006. Emerging roles of pseudokinases. Trends Cell Biol 16: 443-452.

Delon I, Brown NH. 2007. Integrins and the actin cytoskeleton. Curr Opin Cell Biol 19: 43-50.

Flavahan NA, Bailey SR, Flavahan WA, Mitra S, Flavahan S. 2005. Imaging remodeling of the actin cytoskeleton in vascular smooth muscle cells after mechanosensitive arteriolar constriction. Am I Physiol Heart Circ Physiol 288: H660-H669. doi: 10.1152/ajpheart.00608.2004.

Foo SS, Turner CJ, Adams S, Compagni A, Aubyn D, Kogata N, Lindblom P, Shani M, Zicha D, Adams RH. 2006. Ephrin-B2 controls cell motility and adhesion during blood-vessel-wall assembly. Cell 124: 161-173.

Giannone G, Dubin-Thaler BJ, Rossier O, Cai Y, Chaga O, Jiang G, Beaver W, Dobereiner HG, Freund Y, Borisy G, et al. 2007. Lamellipodial actin mechanically links myosin activity with adhesion-site formation. Cell 128: 561-575.

Grashoff C, Aszodi A, Sakai T, Hunziker EB, Fassler R. 2003. Integrinlinked kinase regulates chondrocyte shape and proliferation. $E M B O$ Rep 4: 432-438.

Hao H, Gabbiani G, Bochaton-Piallat ML. 2003. Arterial smooth muscle cell heterogeneity: Implications for atherosclerosis and restenosis development. Arterioscler Thromb Vasc Biol 23: 1510-1520.

Hellstrom M, Kalen M, Lindahl P, Abramsson A, Betsholtz C. 1999. Role of PDGF-B and PDGFR- $\beta$ in recruitment of vascular smooth muscle cells and pericytes during embryonic blood vessel formation in the mouse. Development 126: 3047-3055.

Hynes RO. 2002. Integrins: Bidirectional, allosteric signaling machines. Cell 110: 673-687.

Jakkaraju S, Zhe X, Schuger L. 2003. Role of stretch in activation of smooth muscle cell lineage. Trends Cardiovasc Med 13: 330-335.

Legate KR, Montanez E, Kudlacek O, Fassler R. 2006. ILK, PINCH and parvin: The tIPP of integrin signalling. Nat Rev Mol Cell Biol 7: 20-31.

McDonald PC, Fielding AB, Dedhar S. 2008. Integrin-linked kinaseEssential roles in physiology and cancer biology. I Cell Sci 121: 31213132.

Moiseeva EP. 2001. Adhesion receptors of vascular smooth muscle cells and their functions. Cardiovasc Res 52: 372-386.

Muranyi A, Macdonald JA, Deng JT, Wilson DP, Haystead TA, Walsh MP, Erdodi F, Kiss E, Wu Y, Hartshorne DJ. 2002. Phosphorylation of the myosin phosphatase target subunit by integrin-linked kinase. Biochem J 366: 211-216

Pereira JA, Benninger Y, Baumann R, Goncalves AF, Ozcelik M, Thurnherr T, Tricaud N, Meijer D, Fassler R, Suter U, et al. 2009. Integrin-linked kinase is required for radial sorting of axons and Schwann cell remyelination in the peripheral nervous system. J Cell Biol 185: 147-161.

Sakai T, Li S, Docheva D, Grashoff C, Sakai K, Kostka G, Braun A, Pfeifer A, Yurchenco PD, Fassler R. 2003. Integrin-linked kinase (ILK) is required for polarizing the epiblast, cell adhesion, and controlling actin accumulation. Genes \& Dev 17: 926-940.

Velasco G, Armstrong C, Morrice N, Frame S, Cohen P. 2002. Phosphorylation of the regulatory subunit of smooth muscle protein phosphatase $1 \mathrm{M}$ at Thr850 induces its dissociation from myosin. FEBS Lett 527: 101-104.

Vouret-Craviari V, Boulter E, Grall D, Matthews C, Obberghen-Schilling E. 2004. ILK is required for the assembly of matrix-forming adhesions and capillary morphogenesis in endothelial cells. J Cell Sci 117: 45594569.

Webb RC. 2003. Smooth muscle contraction and relaxation. Adv Physiol Educ 27: 201-206.

Wilson DP, Sutherland C, Borman MA, Deng JT, Macdonald JA, Walsh MP. 2005. Integrin-linked kinase is responsible for $\mathrm{Ca}^{2+}$-independent myosin diphosphorylation and contraction of vascular smooth muscle. Biochem I 392: 641-648.

Zervas CG, Gregory SL, Brown NH. 2001. Drosophila integrin-linked kinase is required at sites of integrin adhesion to link the cytoskeleton to the plasma membrane. J Cell Biol 152: 1007-1018. 


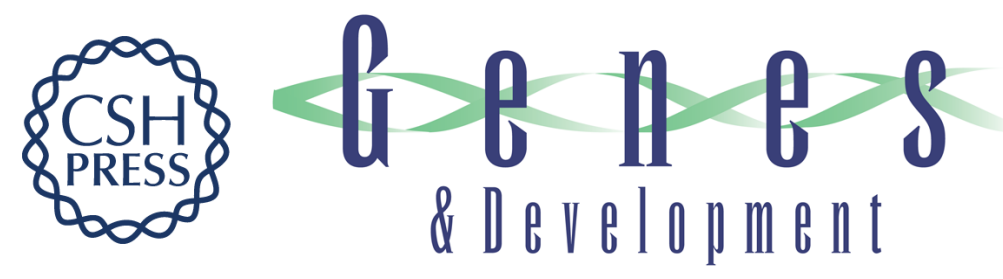

\section{Integrin-linked kinase controls vascular wall formation by negatively regulating Rho/ROCK-mediated vascular smooth muscle cell contraction}

Naoko Kogata, Rachel M. Tribe, Reinhard Fässler, et al.

Genes Dev. 2009, 23:

Access the most recent version at doi:10.1101/gad.535409

Supplemental http://genesdev.cshlp.org/content/suppl/2009/10/02/23.19.2278.DC1

Material

References This article cites 24 articles, 11 of which can be accessed free at: http://genesdev.cshlp.org/content/23/19/2278.full.html\#ref-list-1

License

Email Alerting Receive free email alerts when new articles cite this article - sign up in the box at the top Service right corner of the article or click here.

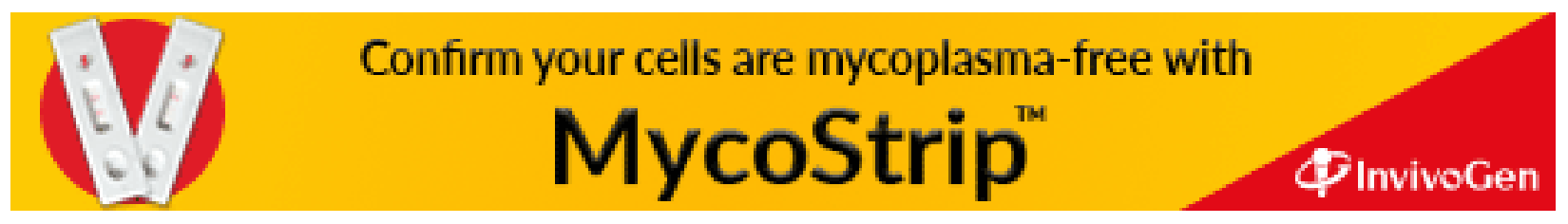

